\title{
ORAL HEALTH LITERACY AMONG ADULTS IN JAKARTA, INDONESIA
}

\author{
Yuniardini Septorini Wimardhani 1,2, Adira Putri Wiryoatmodjo ${ }^{2}$, Cristy Arianta Sitepu2 ${ }^{2}$, Dwiseptia Nadiantari' ${ }^{2}$, \\ Anandina Irmagita Soegyanto ${ }^{1}$, Febrina Rahmayanti', Indriasti Indah Wardhany' ${ }^{1}$, Anton Rahardjo ${ }^{2,3}$, Diah Ayu Maharani ${ }^{3}$ \\ 'Department of Oral Medicine, Faculty of Dentistry, Universitas Indonesia, Depok, Indonesia \\ ${ }^{2}$ Cluster of Clinical and Epidemiology and Clinical Studies in Dentistry, Faculty of Dentistry, Universitas Indonesia, Depok, Indonesia \\ ${ }^{3}$ Department of Preventive and Public Health Dentistry, Faculty of Dentistry, Universitas Indonesia, Jakarta, Indonesia
}

\begin{abstract}
INTRODUCTION: There is an emerging need to improve oral health literacy (OHL) for good oral health outcomes worldwide. Many OHL tools have been developed, and the Indonesian version of Health Literacy in Dentistry (HeLD-ID) has the potential to be a valid and reliable oral health instrument for use in Indonesia.

Овjectives: We aimed to measure OHL using HeLD-ID and to analyze the socioeconomic determinants of OHL among adults in Jakarta.

MATERIAL AND METHODS: In total, 1000 adults representing five regions of Jakarta completed a 29-item OHL self-assessment using the HeLD-ID scale. The self-assessment also gathered socioeconomic and demographic data. The responses were graded on a 5-point Likert-type scale. A higher score indicated better OHL.

RESULTS: The overall mean HeLD-ID score was $2.73 \pm 1.03$. The score was significantly affected by age, region, marital status, the number of dependents, occupation, the level of education, income, and the type of health insurance. Only sex did not affect the scores. The overall Cronbach's a coefficient of the questionnaire was 0.958, and none of the CITC values were $<0.3$.

Conclusion: This study showed that HeLD-ID can successfully be applied to assess the OHL of adults in Jakarta. Additionally, it found that the overall OHL was lower than that of a previous study, with many sociodemographic factors significantly affecting OHL.
\end{abstract}

KEY WORDs: maxillary lateral incisor agenesis, dental anomalies, systematic literature review.

J Stoma 2018; 71, 4: 392-399

DOI: https://doi.org/10.5114/jos.2018.84763

\section{INTRODUCTION}

Oral health literacy (OHL) has become a key issue for oral disease prevention and health promotion [1]. OHL is defined as the capacity to obtain, process, and understand basic oral health information and the services needed to make appropriate oral health decisions [2]. The global burden of oral health problems is not lim- ited to dental diseases, because the problems that result from poor oral health can compromise overall general health and the quality of life [2]. This issue is addressed in the World Health Organization (WHO) Global Oral Health Program, emphasizing the importance of disease prevention and health promotion. The improvement in OHL is one of the priority action items to increase the knowledge and behavior that promote health [1].

\section{JOURNAL OF STOMATOLOGY CZASOPISMO STOMATOLOGICZNE}

ADDRESS FOR CORRESPONDENCE: Yuniardini S. Wimardhani, Department of Oral Medicine, Faculty of Dentistry, Universitas Indonesia, Jl. Salemba Raya No. 4 Jakarta 10430, e-mail: yuniardini@ui.ac.id 
There are many tools available to measure OHL, including the Rapid Estimate of Adult Literacy in Dentistry, the Test of Functional Health Literacy in Dentistry, the Oral Health Literacy Instrument, the Rapid Estimate of Adult Literacy in Medicine and Dentistry, the Comprehensive Measure of Oral Health Knowledge, the Hong Kong Rapid Estimate of Adult Literacy in Dentistry, the Oral Health Literacy Adults Questionnaire, and Health Literacy in Dentistry (HeLD) [1]. A majority of these tools are subjectively biased in terms of word recognition, numeracy, and the reading skills of the respondents. They may also not include other aspects of oral health such as health behavior or service utilization [1]. The more current tools such as HeLD cover more domains while assessing OHL, including communication, access, economic barriers, receptivity, understanding, utilization, and support. HeLD was based on the Health Literacy Management Scale (HeLMS), but it focuses on oral health rather than on overall medical health and is used in different settings globally.

Until our previous study assessed OHL using the Indonesian version of HeLD (HeLD-ID) in a sample population of Indonesian university students and adolescents, data on the OHL of the Indonesian population were unavailable. HeLD-ID has been translated into the Indonesian language using a forward and backward process according to the guidelines for cross-cultural adaptation [3-5]. The results of our previous study indicated that HeLD-ID has the potential to be a valid and reliable oral health instrument to be used in a larger population, particularly in Indonesia. Indonesia is the fourth most populous country worldwide, after China, India, and the United States. Approximately 57\% of Indonesians live on the island of Java, with Jakarta being the most densely populated city in Indonesia with a population of approximately 10 million individuals [6]. It is important to know the OHL of adults in Jakarta to successfully implement programs for the promotion of better health behavior and the prevention of various diseases. The objective of this study was to measure HeLD-ID and to analyze the socioeconomic determinants of OHL among adults in Jakarta.

\section{MATERIAL AND METHODS}

\section{SUBJECTS}

This cross-sectional analytical study was conducted to determine the levels of OHL among adults living in Daerah Khusus Ibukota Jakarta, also called the Special Capital Region of Jakarta, the capital and largest city of Indonesia. The research protocol was approved by the Ethical Committee of the Faculty of Dentistry, Universitas Indonesia. This study was performed in 2015 with 1000 adults representing a total of 9,604,329 individuals from 5 different areas of Jakarta (data from
2010). The respondents were selected using a multistage cluster random sampling method based on a proportional number of people in each area of Jakarta. Informed consent was obtained from every respondent who participated in the study.

\section{MEASURES}

The socioeconomic and demographic data and the data about OHL were collected using a self-administered survey called the HeLD questionnaire. It had been translated into the Indonesian language, validated in our previous study, confirmed, and renamed as HeLD-ID [3, 4]. The original HeLD scale comprised 29 items that were designed to assess OHL [7]. HeLD focused on the difficulty experienced by respondents and comprised seven domains, including receptivity, understanding, support, economic barriers, access, communication, and utilization. The responses were graded on a 5-point Likert-type scale ranging from 0 (unable to do) to 4 (without any difficulty) with scores ranging from 0 to 116 . A higher score indicated better OHL.

\section{STATISTICAL ANALYSIS}

The total HeLD score was calculated based on the mean response for each question. The data obtained were processed using SPSS version 23 for Macintosh. Cronbach's a and corrected item-total correlations (CITCs) were used to assess the internal consistency of the instrument. Alpha, if deleted, was used for optimal subscale assessment and was determined for each item as a measure of overall item consistency. A Cronbach's a coefficient of $\geq 0.70$ was considered as an acceptable level of reliability. If the CITC value was $<0.30$, the item was removed. The normality of the data was determined using the Kolmogorov-Smirnov test. The association among the seven components of HeLDID was measured using Pearson correlation. Various sociodemographic characteristics of the respondents were analyzed for the differences in OHL among the sociodemographic characteristics using the non-parametric Mann-Whitney $U$-test and the non-parametric Kruskal-Wallis test. The significance level was set at 0.05 . Statistically significant differences in the scaled scores with relevant OHL-related factors offered evidence of the instrument's ability to discriminate among the groups.

\section{RESULTS}

All respondents completed the HeLD questionnaire. The overall mean HeLD score for all respondents based on the demographic characteristics was analyzed, and the results are shown in Table 1. The overall mean HeLD score for all 1000 participants was 2.73 with no differ- 
ence in terms of sex, which was the only factor that did not affect the HeLD-ID scores. The respondents located in the central area of Jakarta had the highest HeLD scores compared with those located in the other areas, whereas the respondents living on the islands had the lowest scores. The differences were statistically significant $(p<0.05)$. The respondents in the 18-24-yearold age group had the highest HeLD scores, whereas the $\geq 65$-year-old respondents had the lowest scores. The differences in the scores between the age groups were statistically significant $(p<0.05)$. Their marital status, the number of dependents, occupation, the level of education, income, and the type of health insurance significantly influenced the HeLD score $(p<0.05)$.

Further, the mean of each HeLD domain for the sociodemographic determinants was analyzed. The results are shown in Table 2. The HeLD domain scores were not significantly different in terms of sex. The number of dependents significantly differed for scores in the domains of receptivity, support, economic barrier, and access. The type of health insurance influenced the scores in the domains of receptivity and understanding. The other sociodemographic determinants such as age, marital status, occupa-

TABLE 1. Mean Health Literacy in Dentistry (HeLD) Scores among various sociodemographic characteristics for adult respondents living in the DKI Jakarta area

\begin{tabular}{|c|c|c|c|}
\hline \multirow{2}{*}{ Variable } & \multirow{2}{*}{$n(\%)$} & \multicolumn{2}{|c|}{ HeLD score } \\
\hline & & Mean & SD \\
\hline Total & $1000(100)$ & 2.73 & 1.03 \\
\hline \multicolumn{4}{|l|}{ Gender } \\
\hline Female & $504(50.4)$ & 2.73 & 1.01 \\
\hline Male & $496(49.6)$ & 2.73 & 1.04 \\
\hline \multicolumn{2}{|l|}{$p$-value } & & 0.76 \\
\hline \multicolumn{4}{|l|}{ Area } \\
\hline Central & $323(32.2)$ & 2.99 & 0.95 \\
\hline East & $220(22.0)$ & 2.56 & 1.05 \\
\hline South & 195 (19.5) & 2.81 & 1.04 \\
\hline West & $158(15.8)$ & 2.41 & 1.02 \\
\hline North & $80(8.0)$ & 2.67 & 0.95 \\
\hline Islands & $24(2.4)$ & 2.50 & 1.08 \\
\hline \multicolumn{2}{|l|}{$p$-value } & & $<0.05$ \\
\hline \multicolumn{4}{|l|}{ Age } \\
\hline $18-24$ & 243 & 2.98 & 0.83 \\
\hline $25-44$ & 270 & 2.86 & 0.86 \\
\hline $45-64$ & 270 & 2.75 & 1.05 \\
\hline$>65$ & 217 & 2.28 & 1.23 \\
\hline \multicolumn{3}{|l|}{$p$-value } & $<0.05$ \\
\hline
\end{tabular}

TABLE 1. (Continued)

\begin{tabular}{l|c|c|c|} 
Variable & $n(\%)$ & $\begin{array}{c}\text { HeLD score } \\
\text { Mean }\end{array}$ & SD \\
\hline Marital status \\
\hline Unmarried & $239(23.9)$ & 2.95 & 0.88 \\
\hline Married & $666(66.6)$ & 2.74 & 1.01 \\
\hline Widowed & $95(9.5)$ & 2.11 & 1.21 \\
\hline$p$-value & & & $<0.05$ \\
\hline
\end{tabular}

\begin{tabular}{|c|c|c|c|}
\hline \multicolumn{4}{|c|}{ Number of dependents } \\
\hline None & $357(35.7)$ & 2.60 & 1.11 \\
\hline 1 & 199 (19.9) & 2.67 & 1.02 \\
\hline 2 & $178(17.8)$ & 2.88 & 0.89 \\
\hline 3 & $131(13.1)$ & 2.81 & 1.03 \\
\hline$>3$ & $135(13.5)$ & 2.90 & 0.91 \\
\hline \multicolumn{3}{|c|}{$p$-value } & $<0.05$ \\
\hline
\end{tabular}

\begin{tabular}{l|l|l|l}
\hline Occupation \\
\hline None & 430 & 2.56 & 1.06 \\
\hline Labor worker & 158 & 2.60 & 1.06 \\
\hline Entrepreneur & 207 & 2.81 & 0.94 \\
\hline Private employee & 122 & 3.02 & 0.92 \\
\hline Government employee & 27 & 3.29 & 0.85 \\
\hline Other & 56 & 3.27 & 0.85 \\
\hline$p$-value & & & $<0.05$
\end{tabular}

\begin{tabular}{|c|c|c|c|}
\hline \multicolumn{4}{|l|}{ Level of education } \\
\hline None & 30 & 1.25 & 1.00 \\
\hline $\begin{array}{l}\text { Did not finish } \\
\text { elementary school }\end{array}$ & 56 & 2.21 & 1.23 \\
\hline Elementary school & 183 & 2.21 & 1.08 \\
\hline Middle school & 163 & 2.53 & 0.94 \\
\hline High school & 432 & 2.99 & 0.83 \\
\hline $\begin{array}{l}\text { Diploma/ } \\
\text { Undergraduate/ } \\
\text { Postgraduate }\end{array}$ & 145 & 3.36 & 0.69 \\
\hline \multicolumn{3}{|l|}{$p$-value } & $<0.05$ \\
\hline
\end{tabular}

Income (Rp per month)

\begin{tabular}{l|c|c|c}
\hline$<1$ million & 385 & 2.44 & 1.05 \\
\hline $1-3$ million & 417 & 2.74 & 1.00 \\
\hline$>3$ million & 198 & 3.27 & 0.78 \\
\hline$p$-value & & $<0.05$ \\
\hline
\end{tabular}

\begin{tabular}{|c|c|c|c|}
\hline \multicolumn{4}{|l|}{ Health finance } \\
\hline Government insurance & 730 & 2.94 & 0.90 \\
\hline Private insurance & 59 & 2.98 & 0.89 \\
\hline No insurance & 211 & 2.85 & 0.93 \\
\hline \multicolumn{3}{|l|}{$p$-value } & $<0.05$ \\
\hline
\end{tabular}


TABLE 2. Mean Health Literacy in Dentistry (HeLD) domain scores among various sociodemographic characteristics of adult respondents living in Jakarta area

\begin{tabular}{|c|c|c|c|c|c|c|c|c|c|}
\hline \multirow{2}{*}{ Variable } & \multirow{2}{*}{$n(\%)$} & \multirow{2}{*}{$\begin{array}{c}\text { HeLD } \\
\text { Mean } \pm \text { SD }\end{array}$} & \multicolumn{7}{|c|}{ HeLD Domain } \\
\hline & & & 1 & 2 & 3 & 4 & 5 & 6 & 7 \\
\hline Total & $1000(100)$ & $2.73 \pm 1.03$ & $2.92 \pm 0.91$ & $2.88 \pm 1.32$ & $2.85 \pm 1.22$ & $2.60 \pm 1.30$ & $2.60 \pm 1.30$ & $2.49 \pm 1.27$ & $2.77 \pm 1.25$ \\
\hline \multicolumn{10}{|l|}{ Gender } \\
\hline Female & $504(50.4)$ & $2.73 \pm 1.01$ & $2.91 \pm 0.90$ & $2.88 \pm 1.20$ & $2.85 \pm 1.22$ & $2.60 \pm 1.30$ & $2.60 \pm 1.33$ & $2.46 \pm 1.27$ & $2.79 \pm 1.25$ \\
\hline Male & $496(49.6)$ & $2.73 \pm 1.04$ & $2.94 \pm 0.92$ & $2.94 \pm 1.28$ & $2.82 \pm 1.24$ & $2.58 \pm 1.31$ & $2.60 \pm 1.28$ & $2.52 \pm 1.26$ & $2.74 \pm 1.25$ \\
\hline$p$-value & & 0.76 & 0.35 & 0.34 & 0.39 & 0.51 & 0.74 & 0.50 & 0.46 \\
\hline \multicolumn{10}{|l|}{ Area } \\
\hline Central & $323(32.2)$ & $2.99 \pm 0.95$ & $2.96 \pm 0.85$ & $3.04 \pm 1.08$ & $2.98 \pm 1.08$ & $2.86 \pm 1.14$ & $2.89 \pm 1.15$ & $2.76 \pm 1.10$ & $3.03 \pm 1.05$ \\
\hline East & $220(22.0)$ & $2.56 \pm 1.05$ & $2.99 \pm 0.94$ & $2.74 \pm 1.34$ & $2.74 \pm 1.34$ & $2.36 \pm 1.37$ & $2.38 \pm 1.42$ & $2.29 \pm 1.32$ & $2.54 \pm 1.37$ \\
\hline South & 195 (19.5) & $2.81 \pm 1.04$ & $3.09 \pm 0.91$ & $2.96 \pm 1.20$ & $2.96 \pm 1.20$ & $2.77 \pm 1.36$ & $2.75 \pm 1.28$ & $2.56 \pm 1.33$ & $2.75 \pm 1.32$ \\
\hline West & $158(15.8)$ & $2.41 \pm 1.02$ & $2.66 \pm 0.95$ & $2.60 \pm 1.27$ & $2.60 \pm 1.27$ & $2.31 \pm 1.29$ & $2.25 \pm 1.30$ & $2.53 \pm 1.21$ & $2.48 \pm 1.27$ \\
\hline North & $80(8.0)$ & $2.67 \pm 0.95$ & $2.75 \pm 0.90$ & $2.87 \pm 1.85$ & $2.87 \pm 1.35$ & $2.48 \pm 1.37$ & $2.44 \pm 1.28$ & $2.25 \pm 1.21$ & $2.92 \pm 1.19$ \\
\hline Islands & $24(2.4)$ & $2.50 \pm 1.08$ & $2.97 \pm 0.75$ & $2.76 \pm 1.22$ & $2.76 \pm 1.22$ & $2.15 \pm 1.32$ & $2.28 \pm 1.34$ & $2.25 \pm 1.43$ & $2.70 \pm 1.42$ \\
\hline$p$-value & & $<0.001$ & $<0.001$ & 0.012 & 0.019 & $<0.001$ & $<0.001$ & $<0.001$ & $<0.001$ \\
\hline \multicolumn{10}{|l|}{ Age } \\
\hline $18-24$ & 243 & $2.98 \pm 0.83$ & $3.05 \pm 0.76$ & $3.25 \pm 1.02$ & $3.15 \pm 0.98$ & $2.76 \pm 1.01$ & $2.78 \pm 1.13$ & $2.80 \pm 1.02$ & $3.08 \pm 0.91$ \\
\hline $25-44$ & 270 & $2.86 \pm 0.86$ & $2.85 \pm 0.88$ & $3.20 \pm 1.02$ & $2.93 \pm 1.07$ & $2.66 \pm 1.23$ & $2.74 \pm 1.14$ & $2.66 \pm 1.10$ & $2.94 \pm 1.05$ \\
\hline $45-64$ & 270 & $2.75 \pm 1.05$ & $2.95 \pm 0.95$ & $2.88 \pm 1.34$ & $2.87 \pm 1.26$ & $2.69 \pm 1.29$ & $2.68 \pm 1.32$ & $2.50 \pm 1.27$ & $2.78 \pm 1.27$ \\
\hline$\geq 65$ & 217 & $2.28 \pm 1.23$ & $2.85 \pm 1.03$ & $2.07 \pm 1.56$ & $2.39 \pm 1.43$ & $2.23 \pm 1.53$ & $2.12 \pm 1.52$ & $2.12 \pm 1.52$ & $2.17 \pm 1.55$ \\
\hline$p$-value & & $<0.001$ & $<0.001$ & $<0.001$ & 0.007 & $<0.001$ & $<0.001$ & $<0.001$ & $<0.001$ \\
\hline \multicolumn{10}{|l|}{ Marital status } \\
\hline Not married & $239(23.9)$ & $2.95 \pm 0.88$ & $3.01 \pm 0.81$ & $3.22 \pm 1.07$ & $3.05 \pm 1.04$ & $2.76 \pm 1.11$ & $2.83 \pm 1.14$ & $2.82 \pm 1.05$ & $3.03 \pm 1.00$ \\
\hline Married & $666(66.6)$ & $2.74 \pm 1.01$ & $2.94 \pm 0.92$ & $2.90 \pm 1.31$ & $2.85 \pm 1.25$ & $2.61 \pm 1.32$ & $2.61 \pm 1.30$ & $2.48 \pm 1.27$ & $2.78 \pm 1.24$ \\
\hline Widowed & $95(9.5)$ & $2.11 \pm 1.21$ & $2.62 \pm 1.04$ & $1.90 \pm 1.55$ & $2.36 \pm 1.43$ & $2.14 \pm 1.48$ & $1.97 \pm 1.50$ & $1.72 \pm 1.43$ & $2.00 \pm 1.54$ \\
\hline$p$-value & & $<0.001$ & 0.010 & $<0.001$ & 0.001 & 0.007 & $<0.001$ & $<0.001$ & $<0.001$ \\
\hline \multicolumn{10}{|l|}{ Number of dependents } \\
\hline None & $357(35.7)$ & $2.60 \pm 1.11$ & $2.90 \pm 0.93$ & $2.63 \pm 1.47$ & $2.73 \pm 1.29$ & $2.50 \pm 1.36$ & $2.47 \pm 1.39$ & $2.33 \pm 1.35$ & $2.62 \pm 1.37$ \\
\hline 1 & 199 (19.9) & $2.67 \pm 1.02$ & $2.82 \pm 0.96$ & $2.80 \pm 1.33$ & $2.80 \pm 1.25$ & $2.58 \pm 1.34$ & $2.50 \pm 1.30$ & $2.37 \pm 1.24$ & $2.70 \pm 1.23$ \\
\hline 2 & $178(17.8)$ & $2.88 \pm 0.89$ & $2.94 \pm 0.85$ & $3.16 \pm 1.12$ & $3.06 \pm 1.03$ & $2.78 \pm 1.12$ & $2.76 \pm 1.17$ & $2.69 \pm 1.13$ & $2.93 \pm 1.04$ \\
\hline 3 & $131(13.1)$ & $2.81 \pm 1.03$ & $2.98 \pm 0.87$ & $3.05 \pm 1.20$ & $2.83 \pm 1.24$ & $2.62 \pm 1.36$ & $2.71 \pm 1.28$ & $2.58 \pm 1.27$ & $2.87 \pm 1.25$ \\
\hline$>3$ & $135(13.5)$ & $2.90 \pm 0.91$ & $3.05 \pm 0.87$ & $3.12 \pm 1.14$ & $2.98 \pm 1.16$ & $2.63 \pm 1.26$ & $2.75 \pm 1.21$ & $2.73 \pm 1.18$ & $2.94 \pm 1.16$ \\
\hline$p$-value & & 0.03 & 0.24 & $<0.001$ & 0.09 & 0.49 & 0.10 & 0.003 & 0.05 \\
\hline \multicolumn{10}{|l|}{ Occupation } \\
\hline None & 430 & $2.56 \pm 1.06$ & $2.84 \pm 0.93$ & $2.66 \pm 1.43$ & $2.71 \pm 1.28$ & $2.38 \pm 1.35$ & $2.41 \pm 1.36$ & $2.26 \pm 1.32$ & $2.59 \pm 1.32$ \\
\hline Labor worker & 158 & $2.60 \pm 1.06$ & $2.72 \pm 0.92$ & $2.78 \pm 1.28$ & $2.71 \pm 1.21$ & $2.37 \pm 1.31$ & $2.44 \pm 1.25$ & $2.37 \pm 1.25$ & $2.59 \pm 1.25$ \\
\hline Entrepreneur & 207 & $2.81 \pm 0.94$ & $3.04 \pm 0.84$ & $2.95 \pm 1.32$ & $2.89 \pm 1.22$ & $2.76 \pm 1.28$ & $2.67 \pm 1.25$ & $2.57 \pm 1.18$ & $2.88 \pm 1.21$ \\
\hline Private employee & 122 & $3.02 \pm 0.92$ & $3.05 \pm 0.92$ & $3.29 \pm 0.99$ & $3.18 \pm 1.03$ & $3.03 \pm 1.07$ & $2.93 \pm 1.18$ & $2.84 \pm 1.14$ & $3.08 \pm 1.05$ \\
\hline $\begin{array}{l}\text { Government } \\
\text { employee }\end{array}$ & 27 & $3.29 \pm 0.85$ & $3.32 \pm 0.67$ & $3.46 \pm 0.94$ & $3.38 \pm 0.85$ & $3.23 \pm 1.10$ & $3.28 \pm 1.07$ & $3.12 \pm 1.12$ & $3.25 \pm 1.04$ \\
\hline Others & 56 & $3.27 \pm 0.85$ & $3.27 \pm 0.77$ & $3.40 \pm 0.92$ & $3.19 \pm 0.95$ & $3.10 \pm 0.97$ & $3.19 \pm 1.09$ & $3.16 \pm 1.00$ & $3.26 \pm 0.99$ \\
\hline$p$-value & & $<0.001$ & $<0.001$ & $<0.001$ & $<0.001$ & $<0.001$ & $<0.001$ & $<0.001$ & $<0.001$ \\
\hline
\end{tabular}


TABLE 2. (Continued)

\begin{tabular}{|c|c|c|c|c|c|c|c|c|c|}
\hline Variable & $n(\%)$ & $\begin{array}{c}\text { HeLD } \\
\text { Mean } \pm \text { SD }\end{array}$ & \multicolumn{7}{|c|}{ HeLD Domain } \\
\hline \multicolumn{10}{|l|}{ Level of education } \\
\hline None & 30 & $1.25 \pm 1.00$ & $2.16 \pm 1.20$ & $0.47 \pm 0.99$ & $1.95 \pm 1.39$ & $1.44 \pm 1.58$ & $1.12 \pm 1.39$ & $0.73 \pm 1.04$ & $0.90 \pm 1.20$ \\
\hline $\begin{array}{l}\text { Did not finish } \\
\text { elementary } \\
\text { school }\end{array}$ & 56 & $2.21 \pm 1.23$ & $2.66 \pm 1.13$ & $1.94 \pm 1.58$ & $2.42 \pm 1.51$ & $2.19 \pm 1.54$ & $2.02 \pm 1.47$ & $1.86 \pm 1.51$ & $2.17 \pm 1.57$ \\
\hline $\begin{array}{l}\text { Elementary } \\
\text { school }\end{array}$ & 183 & $2.21 \pm 1.08$ & $2.63 \pm 0.97$ & $2.08 \pm 1.46$ & $2.32 \pm 1.45$ & $2.11 \pm 1.41$ & $1.96 \pm 1.35$ & $1.87 \pm 1.33$ & $2.18 \pm 1.42$ \\
\hline Middle school & 163 & $2.53 \pm 0.94$ & $2.88 \pm 0.88$ & $2.82 \pm 1.27$ & $2.73 \pm 1.28$ & $2.35 \pm 1.34$ & $2.37 \pm 1.28$ & $2.18 \pm 1.23$ & $2.59 \pm 1.22$ \\
\hline High school & 432 & $2.99 \pm 0.83$ & $3.04 \pm 0.81$ & $3.34 \pm 0.93$ & $3.09 \pm 0.97$ & $2.83 \pm 1.13$ & $2.86 \pm 1.13$ & $2.80 \pm 1.04$ & $3.08 \pm 0.97$ \\
\hline $\begin{array}{l}\text { Diploma/ } \\
\text { Undergraduate/ } \\
\text { Postgraduate }\end{array}$ & 145 & $3.36 \pm 0.69$ & $3.26 \pm 0.76$ & $3.50 \pm 0.79$ & $3.30 \pm 0.79$ & $3.23 \pm 0.90$ & $3.43 \pm 0.79$ & $3.30 \pm 0.79$ & $3.41 \pm 0.76$ \\
\hline$p$-value & & $<0.001$ & $<0.001$ & $<0.001$ & $<0.001$ & $<0.001$ & $<0.001$ & $<0.001$ & $<0.001$ \\
\hline \multicolumn{10}{|l|}{ Income } \\
\hline$<1$ million & 385 & $2.44 \pm 1.05$ & $2.78 \pm 0.95$ & $2.57 \pm 1.46$ & $2.63 \pm 1.30$ & $2.21 \pm 1.34$ & $2.25 \pm 1.34$ & $2.12 \pm 1.32$ & $2.46 \pm 1.35$ \\
\hline 1-3 million & 417 & $2.74 \pm 1.00$ & $2.89 \pm 0.93$ & $2.89 \pm 1.29$ & $2.83 \pm 1.20$ & $2.65 \pm 1.26$ & $2.62 \pm 1.26$ & $2.51 \pm 1.21$ & $2.80 \pm 1.20$ \\
\hline$>3$ million & 198 & $3.27 \pm 0.78$ & $3.28 \pm 0.70$ & $3.46 \pm 0.85$ & $3.31 \pm 0.93$ & $3.25 \pm 1.00$ & $3.25 \pm 1.00$ & $3.14 \pm 0.97$ & $3.28 \pm 0.94$ \\
\hline$p$-value & & $<0.001$ & $<0.001$ & $<0.001$ & $<0.001$ & $<0.001$ & $<0.001$ & $<0.001$ & $<0.001$ \\
\hline \multicolumn{10}{|l|}{ Health finance } \\
\hline $\begin{array}{l}\text { Government } \\
\text { insurance }\end{array}$ & 730 & $2.94 \pm 0.90$ & $2.87 \pm 1.31$ & $2.86 \pm 1.20$ & $2.86 \pm 1.20$ & $2.60 \pm 1.27$ & $2.60 \pm 1.28$ & $2.52 \pm 1.35$ & $2.81 \pm 1.22$ \\
\hline Private insurance & 59 & $2.98 \pm 0.89$ & $3.34 \pm 0.88$ & $3.25 \pm 0.85$ & $3.25 \pm 0.85$ & $3.18 \pm 1.03$ & $3.19 \pm 1.07$ & $3.09 \pm 0.86$ & $3.18 \pm 091$ \\
\hline No insurance & 211 & $2.85 \pm 0.93$ & $2.76 \pm 1.46$ & $2.69 \pm 1.33$ & $2.69 \pm 1.33$ & $2.42 \pm 1.43$ & $2.42 \pm 1.39$ & $2.21 \pm 1.35$ & $2.51 \pm 1.39$ \\
\hline$p$-value & & $<0.001$ & 0.39 & 0.84 & 0.026 & 0.001 & $<0.001$ & $<0.001$ & $<0.001$ \\
\hline
\end{tabular}

Legend: 1 - receptivity, 2 - understanding, 3- support, 4-economic barrier, 5-access, 6-communication, 7 - utilization

p-value was tested using Mann-Whitney U-test and Kruskal-Wallis test

HeLD - Health Literacy in Dentistry, SD - standard deviation

tion, the level of education, and income showed significantly different scores for each HeLD domain.

Table 3 shows the mean item scores, Cronbach's a for the subscales if an item was removed, and the CITCs. The Cronbach's a values showed a high relatedness of all items with the overall mean HeLD score, indicating that the consistency of the responses could not be improved by deleting any item. The overall Cronbach's $\alpha$ was 0.958 , with no Cronbach's a for domains with CITC values $<0.7$. None of the CITC values were $<0.3$, indicating that all items could be included in the instrument.

Table 4 shows the correlations among the variables. The item subscale correlations ranged from 0.4 to 0.7 , indicating the multi-dimensionality of the scale.

\section{DISCUSSION}

Oral health inequity exists in Indonesia and in other parts of the world $[8,9]$. The improvement in dental public health is an important issue in Indonesia. Many factors, including sociodemographic determinants, lifestyle, public OHL, oral health service providers, and policy makers, may have contributed to the oral health inequalities. OHL, an emerging public health issue, is seen as an important indicator of oral health outcomes $[10,11]$. It is a health promotion strategy that is included in the five key paths of the $7^{\text {th }}$ Global Conference on Health Promotion of the WHO [12]. Many OHL tools are reported in the literature; however, most of them are heavily biased toward word recognition, numeracy, and reading skills, and they do not address the aspects of health behavior or service utilization [1]. This study used HeLD to assess the OHL of adults in Jakarta. HeLD is based on the Health Literacy Management Scale in Medicine (HeLMS) [13]. Although it was originally developed to assess the OHL among the indigenous population in Australia, the HeLD tool is a promising instrument that is recommended for use in international settings in marginalized and general populations $[7,14]$. It addresses the multidimensional nature of OHL and encompasses the domains of com- 
TABLE 3. Item characteristics and reliability analysis

\begin{tabular}{|c|c|c|c|c|c|}
\hline \multirow{2}{*}{$\begin{array}{l}\text { HeLD } \\
\text { subscale and } \\
\text { items }\end{array}$} & \multicolumn{5}{|c|}{ Reliability } \\
\hline & Mean & SD & $\begin{array}{c}\mathbf{a} \text {, if } \\
\text { deleted }\end{array}$ & CITC & Cronbach's a \\
\hline Receptivity & 2.92 & 0.91 & & & 0.819 \\
\hline HeLD R1 & & & 0.820 & 0.485 & \\
\hline HeLD R2 & & & 0.751 & 0.722 & \\
\hline HeLD R3 & & & 0.755 & 0.708 & \\
\hline HeLD R4 & & & 0.761 & 0.687 & \\
\hline HeLD R5 & & & 0.824 & 0.477 & \\
\hline Understanding & 2.88 & 1.32 & & & 0.931 \\
\hline HeLD U1 & & & 0.930 & 0.822 & \\
\hline HeLD U2 & & & 0.870 & 0.896 & \\
\hline HeLD U3 & & & 0.899 & 0.860 & \\
\hline Support & 2.85 & 1.22 & & & 0.856 \\
\hline HeLDS1 & & & 0.765 & 0.764 & \\
\hline HeLD S2 & & & 0.746 & 0.785 & \\
\hline HeLDS3 & & & 0.877 & 0.644 & \\
\hline Economic barrier & 2.60 & 1.30 & & & 0.889 \\
\hline HeLD E1 & & & 0.854 & 0.769 & \\
\hline HeLD E2 & & & 0.862 & 0.759 & \\
\hline HeLD E3 & & & 0.809 & 0.820 & \\
\hline Access & 2.60 & 1.30 & & & 0.890 \\
\hline HeLD A1 & & & 0.873 & 0.717 & \\
\hline HeLD A2 & & & 0.832 & 0.823 & \\
\hline HeLD A3 & & & 0.833 & 0.820 & \\
\hline HeLD A4 & & & 0.888 & 0.674 & \\
\hline Communication & 2.49 & 1.27 & & & 0.935 \\
\hline HeLD C1 & & & 0.926 & 0.781 & \\
\hline HeLD C2 & & & 0.924 & 0.799 & \\
\hline HeLD C3 & & & 0.922 & 0.825 & \\
\hline HeLD C4 & & & 0.929 & 0.746 & \\
\hline HeLD C5 & & & 0.923 & 0.808 & \\
\hline HeLD C6 & & & 0.926 & 0.779 & \\
\hline HeLD C7 & & & 0.925 & 0.789 & \\
\hline Utilization & 2.77 & 1.25 & & & 0.918 \\
\hline HeLD X1 & & & 0.941 & 0.677 & \\
\hline HeLD X2 & & & 0.879 & 0.853 & \\
\hline HeLD X3 & & & 0.873 & 0.869 & \\
\hline HeLD X4 & & & 0.877 & 0.860 & \\
\hline
\end{tabular}

Cronbach's a for subscales if an item was removed.

CITC - corrected item-total correlation, HeLD - Health Literacy in Dentistry, $S D$ - standard deviation

munication, access, receptivity, understanding, utilization, support, and economic barriers, which impact oral health status [7]. These design features support HeLD's
TABLE 4. Associations (Spearman's correlation) between the seven domains of the Health Literacy in Dentistry (HeLD) scale

\begin{tabular}{|l|c|c|c|c|c|c|c|}
\hline Components & $\mathbf{1}$ & $\mathbf{2}$ & $\mathbf{3}$ & $\mathbf{4}$ & $\mathbf{5}$ & $\mathbf{6}$ & $\mathbf{7}$ \\
\hline Receptivity & - & - & - & - & - & - & - \\
\hline Understanding & 0.416 & - & - & - & - & - & - \\
\hline Support & 0.459 & 0.626 & - & - & - & - & - \\
\hline $\begin{array}{l}\text { Economic } \\
\text { barrier }\end{array}$ & 0.439 & 0.440 & 0.538 & - & - & - & - \\
\hline Access & 0.401 & 0.553 & 0.583 & 0.596 & - & - & - \\
\hline Communication & 0.459 & 0.598 & 0.625 & 0.564 & 0.752 & - & - \\
\hline Utilization & 0.447 & 0.608 & 0.619 & 0.556 & 0.657 & 0.760 & - \\
\hline
\end{tabular}

Correlations were all significant at the $p<0.01$ level. The correlations of the subscale items ranged from 0.4 to 0.7 , indicating the multi-dimensionality of the scale.

role as an appropriate tool to assess the OHL in other parts of the world, particularly in Indonesia $[3,4]$.

The literature shows that poor oral hygiene is a problem among adults in Indonesia [2]. There is an unequal distribution of dentists in Indonesia, and inequity among people gaining access to necessary dental treatment $[15,16]$. Previous studies on OHL using HeLD-ID among undergraduate students encouraged further validation of HeLD-ID in a general sample population [4]. The current study among adults from Jakarta, the capital city of Indonesia, includes many people from different parts of Indonesia. The presence of inequity in terms of many sociodemographic aspects of society is still a challenge for Jakarta [6]. Although Indonesia comprises many islands with numerous tribes with different languages and dialects, the results of this study provide information that may increase OHL and better oral health among all residents of Jakarta. Further studies should be conducted to explore the instrument's applicability in other Indonesian settings.

This study utilizes the previously translated and validated Indonesian version of HeLD [3] to evaluate the OHL in community settings, including 1000 people from Jakarta. Generalization is limited by the fact that it may not be representative of all Indonesian adults. The Cronbach's a for HeLD-ID was 0.95 , indicating a strong internal consistency. On assessing each item of the questionnaire, the first question of the reliability subscale showed a low CITC reliability score, indicating that there is a gap between the question and the HeLD scale in general, which possibly occurred because of the cross-cultural adaptation of the questionnaire.

The overall OHL of the adults in this study is lower than that of the undergraduate students in our previous study [3,4]. Despite other previous studies that show females have better OHL than males, our study showed that male and female adults have the same level of OHL $[3,4]$, which matches the results of an In- 
dian study [16]. Both sexes showed the lowest score in the communication domain, a domain that measures the ability to communicate with the dentist to gain information related to oral health. The location of the respondents within Jakarta may have influenced the HeLD score. The HeLD-ID scores varied in terms of the area of Jakarta where the respondent lives, with statistically significant differences. The people living in the western area, in the islands, and the eastern part of Jakarta had lower HeLD scores and the highest number of social problems [6]. The HeLD scores also showed statistically significant differences $(p<0.05)$ in terms of age, marital status, the number of dependents, occupation, the level of education, income, and the type of health insurance. The results of this study are consistent with those of a few previous studies reporting that the factors influencing the use of dental health services are age, education, and education level [18]. The OHL scores tend to decrease with age, which is consistent with the result of a study conducted in Canada showing that age and aging are determinants of health literacy [18]. Older people tend to have lower health literacy levels due to factors such as mental health, reading ability, health status, and vision. The differences in OHL among the elderly are found in the domains of understanding, support, communication, and utilization. All four domains show a decrease in OHL levels as age increases. There was no significant difference in the domains of receptivity, access, and economic barrier. The low OHL levels in the domain of understanding may be caused by a decrease in cognitive functions among the elderly, the difficulties in gaining access to new information, and oral health related materials that use unfamiliar or complex terminology that is difficult to understand $[18,19]$. The HeLD score in the current study is also statistically different $(p<0.05)$ in terms of age, marital status, the number of dependents, the type of occupation, the level of education, income, and the type of health insurance. The individuals who were single had better HeLD scores compared with those of widowers. Single individuals may be more independent about seeking information related to dental care. However, an analysis of the association between OHL and oral health outcome is needed. The results of the current study can be the foundation for policy development and public health program planning, particularly designed to improve the $\mathrm{OHL}$ based on related and specific domains, since OHL is not a permanent skill but may decrease due to a variety of factors [18].

\section{CONCLUSIONS}

To the best of our knowledge, this study is the first to report about OHL in a sample of a large population of Jakarta. The results show that HeLD-ID can be imple- mented in the larger population of Indonesia, particularly in Jakarta. The results show that the OHL of adults in Jakarta is lower than that of undergraduate students. The results of this study can be the foundation to develop policies and plan public health programs, particularly designed to improve OHL based on the various factors influencing that skill.

\section{ACKNOWLEDGEMENTS}

This work was supported by a Universitas Indonesia Research Grant. The authors are grateful for the support given from the Government of the Special Region of Jakarta, Indonesia.

\section{CONFLICT OF INTEREST}

The authors declare no potential conflicts of interest with respect to the research, authorship, and/or publication of this article.

\section{References}

1. Dickson-Swift V, Kenny A, Farmer J, et al. Measuring oral health literacy: A scoping review of existing tools. BMC Oral Health 2014; 14: 148.

2. Petersen PE, Ogawa $\mathrm{H}$. The global burden of periodontal disease: toward integration with chronic disease prevention and control. Periodontol 2000 2012; 60: 15-39.

3. Rahardjo A, Wachid MN, Adiatman M, et al. Health literacy in dentistry among undergraduate students in Indonesia. Asian J Epidemiol 2015; 9: 24-29.

4. Rahardjo A, Adinda S, Nasia AA, et al. Oral health literacy in Indonesian adolescent. J Int Dent Med Res 2015; 8: 1-5.

5. Wong HM, Bridges SM, Yiu CKY, et al. Development and validation of Hong Kong rapid estimate of adult literacy in dentistry. J Investig Clin Dent 2012; 3: 118-127.

6. Jakarta Center of Statistics Bureau 2017 (BPS-Statistics of DKI Jakarta Province). Jakarta in Figures. 2017. ISSN: 0215.2150.

7. Jones K, Parker E, Mills H, et al. Development and psychometric validation of a Health Literacy in Dentistry scale (HeLD). Community Dent Health 2014; 31: 37-43.

8. Mejia GC, Parker EJ, Jamieson LM. An introduction to oral health inequalities among Indigenous and non-Indigenous populations. Int Dent J 2010; 60 (3 Suppl 2): 212-215.

9. Rahardjo A, Maharani DA. A review of Indonesia's dental health - past, present, and future. Int J Clin Prev Dent 2014; 10: 121-126.

10. Horowitz AM, Kleinman DV. Oral health literacy: The new imperative to better oral health. Dent Clin North Am 2008; 52: 333-344.

11. Horowitz AM, Kleinman DV. Oral health literacy: A pathway to reducing oral health disparities in Maryland. J Public Health Dent 2012; 72: S26-S30.

12. Petersen PE, Kwan S. The 7th WHO Global conference on health promotion - toward integration of oral health. Community Dent Health 2010; 27 (Suppl 1): 129-136.

13. Jordan JE, Buchbinder R, Briggs AM, et al. The Health Literacy Management Scale (HeLMS): A measure of an individual's capacity to seek, understand, and use health information within the healthcare setting. Patient Educ Couns 2013; 91: 228-235.

14. Parker EJ, Misan G, Chong A, et al. An oral health literacy intervention for Indigenous adults in a rural setting in Australia. BMC Public Health 2012; 12: 461.

15. Maharani DA. Inequity in dental care utilization in the Indonesian population with a self-assessed need for dental treatment. Tohoku J Exp Med 2009; 218: 229-239. 
16. Maharani DA. Do the Indonesians receive the dental care treatment they need? A secondary analysis on self-perceived dental care need. ISRN Dent 2012; 2012: 769809.

17. Haridas RSS, Ajagannanavar S, Tikare S, et al. Oral health literacy and oral health status among adults attending dental college hospital in India. J Int Oral Health 2014; 6: 61-66.

18. Rootman I, Ronson B. Literacy and health research in Canada where have we been and where should we go? Can J Public Health 2005; 96 Suppl 2: S62-S77.

19. D'Cruz AM, Shankar Aradhya MR. Health literacy among Indian adults seeking dental care. Dent Res J (Isfahan) 2013; 10: 20-24. 